Revista Brasileira de Fisiologia do Exercício 2018;17(2):86-92

\title{
ARTIGO ORIGINAL \\ Características da aptidão aeróbia e neuromuscular de praticantes dos projetos de extensão de uma instituição de ensino superior em Campo Grande/MS \\ Characteristics of aerobic and neuromuscular fitness of acadêmicos of a higher education institution in Campo Grande/MS
}

\author{
William Eiji Miyagi", Gabriel Elias Ota**, Camila Souza de Morais ${ }^{* * *}$, Cleberson Dias Lima**, \\ Fernando Sérgio Silva Barbosa ${ }^{* * * *}$, Leonardo Emmanuel de Medeiros Lima ${ }^{* * * *}$, Geovany \\ Rafael Bisol ${ }^{\star \star * * * *}$
}

*Discente da Faculdade Unigran Capital, Campo Grande/MS, **Docente da Faculdade Estácio de Sá, Campo Grande/MS, ${ }^{* * *}$ Mestre em Saúde e Sociedade PPGSD/UFMS, ${ }^{* * *}$ Docente da Universidade Federal de Rondônia (UNIR), ${ }^{* * * * * D o c e n t e ~ d a ~ U n i v e r s i d a d e ~ A n h e m b i ~ M o r u m b i, ~}$ $S P,{ }^{* * * * * D o c e n t e ~ d a ~ F a c u l d a d e ~ U n i g r a n ~ C a p i t a l, ~ C a m p o ~ G r a n d e / M S ~}$

Recebido em 13 de janeiro de 2018; aceito em 20 de junho de 2018.

Endereço para correspondência: Geovany Rafael Bisol, Faculdade Unigran Capital, Rua Abrão Júlio Rahe, 325, 79010-010 Campo Grande MS, E-mail: prof.geovanybisol@gmail.com; William Eiji Miyagi: wemiyagi@hotmail.com; Gabriel Elias Ota: gabriel.elias.ota@gmail.com; Camila Souza de Morais: morais.camila@outlook.com; Cleberson Dias Lima: cleberson07@hotmail.com; Fernando Sérgio Silva Barbosa: fernandossb@hotmail.com; Leonardo Emmanuel de Medeiros Lima: leonardolimadocente@gmail.com

\section{Resumo}

O objetivo do estudo foi verificar as características da aptidão aeróbia e neuromuscular de participantes dos projetos de extensão de uma instituição de ensino superior da cidade de Campo Grande/MS. O presente estudo foi de caráter quantitativo e descritivo. Os dados de 28 indivíduos (homens: $27 \pm 11$ anos; $74,8 \pm 7,9 \mathrm{~kg}$ de massa corporal e $171 \pm 7,1 \mathrm{~cm}$ de estatura; mulheres: $32 \pm 14$ anos; $66,9 \pm 14,1 \mathrm{~kg}$ de massa corporal e $161,4 \pm 8,9 \mathrm{~cm}$ de estatura) foram extraídos do banco de dados de uma instituição de ensino superior. Para verificar o perfil da aptidão aeróbia foram utilizados os resultados da avaliação do limiar anaeróbio estimado por meio da determinação do ponto de deflexão da frequência cardíaca observado em teste incremental. A análise do perfil da aptidão neuromuscular foi realizada a partir dos resultados dos testes de flexão de braços e flexão de tronco. Os resultados da avaliação do limiar anaeróbio foram de $8,9 \pm 0,4 \mathrm{~km} \cdot \mathrm{h}-1$. No teste de flexão de tronco, os homens realizaram 30,8 $\pm 10,8$ repetições e as mulheres $21,3 \pm 6,2$ repetições. No teste de flexão de braços, os homens realizaram $20,5 \pm 12,1$ repetições e as mulheres $19,7 \pm 7,9$ repetições. Nos homens foi observado que $40 \%$ dos indivíduos foram classificados como fracos e $20 \%$ como abaixo da média na avaliação da aptidão neuromuscular, tanto no teste de flexão de tronco quanto de flexão de braços. As mulheres apresentaram prevalência de $40 \%$ na classificação fraco para flexão de tronco, e $46,2 \%$ na classificação média para o teste de flexão de braços. Assim, o perfil dos praticantes dos projetos de extensão de uma instituição de ensino superior de Campo Grande/MS sugere uma baixa aptidão física voltada para a saúde, indicando a necessidade de ações e planejamentos de programas que procurem desenvolver a aptidão física desses indivíduos.

Palavras-chave: frequência cardíaca, aptidão física, exercício.

\section{Abstract}

The objective of the study was to verify the characteristics of the aerobic and neuromuscular fitness of participants of extension projects of an education institution in the city of Campo Grande/MS. The present study was quantitative and descriptive. The data of 28 individuals (men: $27 \pm 11$ years, body mass $74,8 \pm 7,9 \mathrm{~kg}$ and height $171 \pm 7,1 \mathrm{~cm}$, women: $32 \pm 14$ years, body mass $66,9 \pm 14,1 \mathrm{~kg}$ and height $161,4 \pm 8,9 \mathrm{~cm}$ ) were extracted from the database of an education institution. In order to verify the aerobic fitness profile, the results of the estimated anaerobic threshold were determined by means of determination of the heart rate deflection point observed in an incremental test. The analysis of the neuromuscular fitness profile was performed from the results of the arms flexion and trunk flexion tests. The results of the 
anaerobic threshold assessment were $8.9 \pm 0.4 \mathrm{~km} . \mathrm{h}-1$. In the trunk flexion test, the men performed $30.8 \pm 10.8$ repetitions and the women performed $21.3 \pm 6.2$ repetitions. In the arm flexion test, men performed $20.5 \pm 12.1$ repetitions and women $19.7 \pm 7.9$ repetitions. In the men we observed that $40 \%$ of the individuals were classified as weak and $20 \%$ as below the average in the assessment of neuromuscular fitness, both in the trunk flexion test and in the arm flexion test. The women presented a prevalence of $40 \%$ in the weak classification for trunk flexion and $46.2 \%$ in the average classification for the arm flexion test. Thus, the profile of practitioners of extension projects at an education institution in Campo Grande/MS suggests a low physical fitness for health, indicating the need for actions and planning of programs that seek to develop the physical fitness of these individuals.

Key-words: heart rate, physical fitness, exercise.

Introdução

A prática regular de exercícios físicos promove diversos benefícios à saúde [1-7]. Esses efeitos benéficos podem ser observados desde os anos iniciais até a vida adulta e no período de envelhecimento [8]. Durante os anos iniciais, a disciplina de Educação Física, presente nos currículos da educação básica, permite o desenvolvimento das capacidades físicas, habilidades motoras básicas, experiências iniciais à prática de esportes, conhecimento sobre jogos, brincadeiras, além de diversos aspectos sociais e atitudinais [9].

No início da vida adulta, esses jovens podem buscar a prática regular de exercícios físicos em atividades que tragam prazer e satisfação [10]. Especificamente, jovens que ingressam no ensino superior podem ter acesso a diferentes atividades físicas e modalidades esportivas oferecidas pelas instituições de Ensino Superior por meio de projetos de extensão [2], sejam elas voltadas para saúde e estética ou para fins de competição. Os exercícios físicos ou treinamentos esportivos em qualquer uma dessas etapas da vida segue uma série de princípios que podem permitir o bem-estar físico, mental e social, assim como otimizar os efeitos benéficos e objetivos propostos [11].

Os praticantes dessas atividades/modalidades dos projetos de extensão podem ser praticantes experientes devido à prática ao longo da vida ou podem ser indivíduos iniciantes e que tiveram uma prática regular de atividade física. Além disso, programas de exercícios físicos e esportivos oferecidos por instituições superiores podem apresentar frequentadores com diferentes níveis de aptidão física, pois atendem toda a comunidade externa. Sendo assim, avaliações físicas com esses praticantes são essenciais para estabelecer perfil desses sujeitos e o nível de desempenho.

Estabelecer o perfil físico de indivíduos submetidos à prática de exercícios físicos é um dos aspectos mais importantes para os profissionais de Educação Física em relação ao treinamento. As avaliações permitem conhecer as capacidades individuais do aluno/atleta, fazendo com que seja possível planejar com excelência todo o programa de treinamento, prescrever intensidades ideais de exercício em acordo com os objetivos propostos, estabelecer objetivos plausíveis, verificar e otimizar os resultados [12,13]. Ainda mais, esses resultados podem ser analisados de modo longitudinal e também podem ser comparados com resultados encontrados na literatura para populações semelhantes, permitindo realizar análises sobre 0 perfil de desempenho desses alunos e estabelecer as estratégias para o planejamento do treinamento.

Estudantes universitários e todos os praticantes dos projetos oferecidos pelas instituições de ensino superior podem apresentar diferentes padrões de composição corporal e de desempenho físico como as capacidades físicas de força, resistência muscular localizada e aptidão aeróbia. Sendo assim, faz-se necessário investigar qual o perfil físico desses indivíduos.

No contexto atual, muitos indivíduos não mantêm uma prática regular de exercícios físicos. As avaliações físicas podem contribuir para verificar o estado de treinamento e o perfil da composição corporal, que permitem estabelecer os objetivos específicos e verificar os resultados ao longo da realização dos projetos. Até mesmo nas atividades de treinamento desportivo, esses indivíduos muitas vezes não mantêm os hábitos de alimentação e treinamento durante os períodos de recesso acadêmico, que acaba influenciando na manutenção dos resultados alcançados no desempenho e na aptidão física.

Portanto, estabelecer o perfil físico desses participantes pode contribuir para 0 planejamento das atividades realizadas nesses projetos e também para nortear as ações de incentivo à prática dessas atividades pelas instituições que as oferecem. Sendo assim, o objetivo do presente estudo foi caracterizar o perfil da aptidão aeróbia e neuromuscular de 
praticantes dos projetos de extensão de uma instituição de ensino superior na cidade de Campo Grande/MS.

\section{Material e métodos}

O presente estudo foi de caráter quantitativo e descritivo. O local de pesquisa foi uma instituição de ensino superior da cidade de Campo Grande/MS. Todos os testes foram realizados pelos integrantes do laboratório de avaliação física da instituição, que eram acadêmicos de Educação Física do último ano de graduação.

Foram selecionados os dados de 11 indivíduos do sexo masculino e 17 do sexo feminino, participantes de projetos de extensão voltados à prática de exercícios físicos e modalidades esportivas de uma instituição de ensino superior. Alguns indivíduos não apresentavam, em seu banco de dados, todos os testes de aptidão física devido a restrições médicas ou por outros motivos, porém os dados existentes foram analisados.

Os dados utilizados para o presente estudo foram fornecidos pelo laboratório de avaliação física da instituição, com autorização prévia documentada. Todas as avaliações foram realizadas por estudantes capacitados que integram o laboratório. As identidades dos sujeitos foram mantidas em sigilo e somente tiveram acesso às informações e aos resultados dos testes os pesquisadores envolvidos na pesquisa e o próprio indivíduo.

\section{Antropometria}

Para caracterização dos sujeitos do estudo, foram selecionados os dados da massa corporal, que foi realizada por meio de uma balança digital, enquanto que a estatura foi mensurada por meio de um estadiômetro.

\section{Avaliação da aptidão aeróbia}

A avaliação da aptidão aeróbia foi realizada por meio da determinação do limiar anaeróbio. Os indivíduos foram submetidos ao teste incremental com intensidade inicial de 6 a $8 \mathrm{~km} \cdot \mathrm{h}-1$ e incrementos de $1 \mathrm{~km} \cdot \mathrm{h}-1$ a cada estágio de 2 minutos até a exaustão. A frequência cardíaca (FC) foi mensurada durante todo o teste por um cinto transmissor (T31, Polar Electro, Kempele, Finlândia) e o valor observado ao final de cada estágio correspondeu à intensidade de exercício.

O limiar anaeróbio foi determinado por meio da identificação do ponto de deflexão da FC, seguindo as recomendações de Conconi et al. [14]. O ponto de deflexão da FC foi determinado pelo método visual, utilizando o ponto de interseção entre duas retas provenientes de ajustes lineares com os pontos da relação intensidade-FC antes do ponto de deflexão e após [15].

Somente os indivíduos que apresentavam em seu banco dados o teste incremental e/ou apresentaram dados suficientes de FC e intensidade de exercício foram avaliados quanto à determinação do LAn. Sendo assim, quando não foi possível determinar o ponto de deflexão da FC ou quando não constavam resultados dessa variável, o indivíduo foi excluído da análise da aptidão aeróbia.

\section{Teste de força-resistência abdominal e flexão de braços}

Os indivíduos iniciaram o teste em decúbito dorsal, com os joelhos flexionados a $90^{\circ} \mathrm{e}$ os braços cruzados sobre o tórax. O sujeito realizou a flexão do tronco até que os cotovelos encostassem nas coxas e depois retornava à posição inicial.

O indivíduo foi instruído a realizar o maior número possível de flexões em um minuto.

Para o teste de flexão de braços o indivíduo posicionou-se com as palmas das mãos e ponte dos pés no solo em decúbito ventral, com os cotovelos estendidos. O avaliado foi instruído a executar a flexão do cotovelo até que o tórax se aproximasse do solo e então retornava à posição inicial. $O$ sujeito foi instruído a realizar o número máximo de repetições no período de 1 minuto. Nos indivíduos do sexo feminino, os apoios incluíram os joelhos.

Para classificação dos indivíduos quanto ao desempenho nos testes de abdominal e de flexão de braços foram utilizados os termos fraco, abaixo da média, média, acima da média, excelente, seguindo uma tabela de referência [16]. 
Tabela I - Valores de referência para o teste de flexão de braços para homens e mulheres.

Classificação para homens ( $\mathrm{n}^{\circ}$ de repetições por minuto)

\begin{tabular}{|c|c|c|c|c|c|}
\hline Idade & Excelente & Acima da média & Média & Abaixo da média & Fraco \\
\hline $15-19$ & $\geq 39$ & 29 a 33 & 23 a 28 & 13 a 22 & $\leq 17$ \\
\hline $20-29$ & $\geq 36$ & 29 a 35 & 22 a 28 & 17 a 21 & $\leq 16$ \\
\hline $30-39$ & $\geq 30$ & 22 a 29 & 17 a 21 & 12 a 16 & $\leq 11$ \\
\hline $40-49$ & $\geq 22$ & 17 a 21 & 11 a 16 & 10 a 12 & $\leq 09$ \\
\hline $50-59$ & $\geq 21$ & 13 a 12 & 10 a 12 & 07 a 09 & $\leq 06$ \\
\hline $60-69$ & $\geq 18$ & 11 a 17 & 08 a 10 & 05 a 07 & $\leq 04$ \\
\hline \multicolumn{6}{|c|}{ Classificação para mulheres ( ${ }^{\circ}$ de repetições por minuto) } \\
\hline Idade & Excelente & Acima da média & Média & Abaixo da média & Fraco \\
\hline $15-11$ & $\geq 33$ & 25 a 32 & 18 a 24 & 12 a 17 & $\leq 11$ \\
\hline $20-29$ & $\geq 30$ & 2129 & 15 a 20 & 10 a 14 & $\leq 9$ \\
\hline $30-39$ & $\geq 27$ & 20 a 26 & 13 a 19 & 08 a 12 & $\leq 7$ \\
\hline $40-49$ & $\geq 24$ & 15 a 23 & 11 a 14 & 05 a 10 & $\leq 4$ \\
\hline $50-59$ & $\geq 21$ & 11 a 21 & 07 a 10 & 02 a 06 & $\leq 1$ \\
\hline $60-69$ & $\geq 17$ & 12 a 16 & 05 a 11 & 02 a 04 & $\leq 1$ \\
\hline
\end{tabular}

Fonte: Fernandes [16].

Tabela II - Valores de referência para o teste de flexão de tronco (abdominal) para homens e mulheres.

\begin{tabular}{|c|c|c|c|c|c|}
\hline \multicolumn{6}{|c|}{ Classificação para homens ( $n^{\circ}$ de repetições por minuto) } \\
\hline Idade & Excelente & Acima da média & Média & Abaixo da média & Fraco \\
\hline $15-19$ & $\geq 48$ & 42 a 47 & 38 a 41 & 33 a 37 & $\leq 32$ \\
\hline $20-29$ & $\geq 43$ & 37 a 42 & 33 а 36 & 29 a 32 & $\leq 18$ \\
\hline $30-39$ & $\geq 36$ & 31 a 35 & 27 a 30 & 22 a 26 & $\leq 21$ \\
\hline $40-49$ & $\geq 26$ & 26 a 30 & 22 a 25 & 17 a 21 & $\leq 16$ \\
\hline $50-59$ & $\geq 23$ & 22 a 25 & 18 a 21 & 13 a 17 & $\leq 12$ \\
\hline $60-69$ & $\geq 23$ & 17 a 22 & 12 a 16 & 07 a 11 & $\leq 6$ \\
\hline \multicolumn{6}{|c|}{ Classificação para mulheres ( $n^{\circ}$ de repetições por minuto) } \\
\hline Idade & Excelente & Acima da média & Média & Abaixo da média & Fraco \\
\hline $15-11$ & $\geq 42$ & 16 a 41 & 32 a 35 & 27 a 31 & $\leq 26$ \\
\hline $20-29$ & $\geq 36$ & 3135 & 25 a 30 & 21 a 24 & $\leq 20$ \\
\hline $30-39$ & $\geq 29$ & 24 a 23 & 20 a 23 & 15 a 19 & $\leq 14$ \\
\hline $40-49$ & $\geq 25$ & 20 a 24 & 15 a 19 & 07 a 14 & $\leq 6$ \\
\hline $50-59$ & $\geq 19$ & 12 a 13 & 05 a 11 & 03 a 04 & $\leq 2$ \\
\hline $60-69$ & $\geq 16$ & 12 a 12 & 04 a 11 & 02 a 03 & $\leq 1$ \\
\hline
\end{tabular}

Fonte: Fernandes [16].

Análise estatística

Os dados foram apresentados em média \pm desvio padrão. Os resultados da aptidão neuromuscular (flexão de tronco e flexão de braços) foram apresentados quanto ao sexo e valores percentuais para a prevalência em cada classificação. Para realizar a análise descritiva das variáveis foi utilizado o software SPSS versão 16.0 (SPSS Inc., Chicago, IL, EUA).

Resultados e discussão

A idade, massa corporal total e estatura dos sujeitos estão apresentadas na Tabela III.

Tabela III - Média \pm desvio padrão das variáveis de idade, massa corporal e estatura dos participantes do estudo.

\begin{tabular}{llll}
\hline & Idade (anos) & Massa corporal $(\mathrm{kg})$ & Estatura $(\mathrm{cm})$ \\
\hline Homens $(\mathrm{N}=11)$ & $27 \pm 11$ & $74,8 \pm 7,9$ & $171,0 \pm 7,1$ \\
Mulheres $(\mathrm{N}=17)$ & $32 \pm 14$ & $66,9 \pm 14,1$ & $161,4 \pm 8,9$ \\
Total $(\mathrm{N}=28)$ & $30 \pm 13$ & $70,0 \pm 12,5$ & $165,2 \pm 9,4$ \\
\hline
\end{tabular}

Fonte: Dados de pesquisa de campo. 
Tabela IV - Resultados dos testes de flexão de tronco (abdominal) e flexão de braços.

\begin{tabular}{lll} 
& $\begin{array}{l}\text { Flexão de tronco } \\
\text { (Repetições em 1 minuto) }\end{array}$ & $\begin{array}{l}\text { Flexão de braços } \\
\text { (Repetições em 1 minuto) }\end{array}$ \\
\hline Homens & $30,8 \pm 10,8$ & $20,5 \pm 12,1$ \\
& $(\mathrm{~N}=10)$ & $(\mathrm{N}=10)$ \\
Mulheres & $21,3 \pm 6,2$ & $19,7 \pm 7,9$ \\
& $(\mathrm{~N}=15)$ & $(\mathrm{N}=13)$ \\
Total & $25,1 \pm 9,4$ & $20,0 \pm 9,7$ \\
& $(\mathrm{~N}=25)$ & $(\mathrm{N}=23)$
\end{tabular}

Fonte: Dados de pesquisa de campo.

A quantidade de abdominais realizada em 1 minuto e de flexões de braços está apresentada na Tabela IV.

A Tabela $V$ apresenta as classificações dos valores observados para os testes de flexão de braços e flexão de tronco (abdominal). Para os homens, no teste de flexão de braços e flexão de troncos foram observados maiores valores para resultados de indivíduos classificado como fracos $(40,0 \%)$. Nas mulheres, foi observado maior prevalência na classificação fraco para o teste de flexão de tronco (40\%), enquanto que no teste de flexão de braços foi observado uma maior prevalência na classificação média (46,2\%).

Tabela V - Resultados das avaliações do teste de aptidão neuromuscular (teste de flexão de tronco e flexão de braços).

\begin{tabular}{llllll}
\hline & $\begin{array}{l}\text { Excelente } \\
(\%)\end{array}$ & $\begin{array}{l}\text { Acima da média } \\
(\%)\end{array}$ & $\begin{array}{l}\text { Média } \\
(\%)\end{array}$ & $\begin{array}{l}\text { Abaixo da média } \\
(\%)\end{array}$ & $\begin{array}{l}\text { Fraco } \\
(\%)\end{array}$ \\
\hline $\begin{array}{l}\text { Homens } \\
\text { Flexão de tronco } \\
(\mathrm{N}=10)\end{array}$ & 20,0 & 10,0 & 10,0 & 20,0 & 40,0 \\
$\begin{array}{l}\text { Flexão de braços } \\
(\mathrm{N}=10)\end{array}$ & 20,0 & 10,0 & 10,0 & 20,0 & 40,0 \\
$\begin{array}{l}\text { Mulheres } \\
\begin{array}{l}\text { Flexão de tronco } \\
(\mathrm{N}=15)\end{array}\end{array}$ & 6,7 & 20,0 & & & 40,0 \\
$\begin{array}{l}\text { Flexão de braços } \\
(\mathrm{N}=13)\end{array}$ & 23,1 & 7,7 & 26,7 & 6,7 & 0,0 \\
$\begin{array}{l}\text { Total } \\
\text { Abdominal }(\mathrm{n}=25)\end{array}$ & 12 & 16 & 46,2 & 23,1 & \\
$\begin{array}{l}\text { Flexão de braços } \\
(\mathrm{n}=23)\end{array}$ & 21,7 & 8,7 & & & 40 \\
\hline Fonte: Dados de pesquisa de campo. & & 20 & 12 & 17,4 \\
\hline
\end{tabular}

Os resultados observados no presente estudo em relação à avaliação da resistência muscular demonstraram que uma considerável parte dos indivíduos apresentaram valores abaixo da média (Tabela V). Esses resultados corroboram os achados de Glaner et al. [17], que verificaram em universitários da Universidade Federal de Santa Maria/RS, uma precária aptidão física voltada para a saúde. Ainda mais, similar aos achados do presente estudo, os autores verificaram uma grande heterogeneidade na amostra, observando valores de excelência nesses sujeitos.

Em outro estudo, Loch et al. [18] verificaram que estudantes universitários da disciplina de Educação Física Curricular apresentaram pequena proporção para baixa aptidão nos testes de resistência muscular $(21,0 \%$ para abdominal em 1 minuto e $25,8 \%$ para o teste de flexão/extensão de braços), mas outros índices associados à aptidão física voltada para saúde como flexibilidade de tronco e consumo máximo de oxigênio, apresentaram uma maior proporção para a classificação de baixa aptidão.

Nestes dois trabalhos [17,18], os autores destacaram os baixos níveis de aptidão física voltada para saúde, sugerindo e alertando sobre a necessidade de implementação e elaboração de programas de atividade física voltadas para a saúde. Outros estudos que investigaram a aptidão física relacionada à saúde também reportaram achados semelhantes ao presente estudo, reforçando os baixos níveis de aptidão física desses indivíduos $[19,20]$. É 
importante ressaltar que no presente estudo, a amostra incluiu indivíduos que não são, necessariamente, universitários. Os projetos de extensão atendem também sujeitos da comunidade externa, que pode ter influenciado nos resultados observados.

Em relação à avaliação da aptidão aeróbia, no presente estudo foram obtidos os valores correspondentes a nove sujeitos. Os valores de LAn foram de 8,9 $\pm 0,4 \mathrm{~km} \cdot \mathrm{h}-1$, enquanto que a FC correspondente ao LAn foi de $181 \pm 35 \mathrm{bpm}$.

Ao avaliar corredores treinados de nível regional, Brunetta et al. [21] observaram valores para o LAn determinado por meio do ponto de deflexão da FC de 11,4 $\pm 0,8 \mathrm{~km} \cdot \mathrm{h}-1$. Em corredores de elite, Kjertakov et al. [22] verificaram um LAn correspondente a 15,6 $\pm 0,7$ $\mathrm{km} \cdot \mathrm{h}-1$. Já para homens saudáveis, Nascimento et al. [15] encontraram um valor de 9,2 $\pm 1,3$ $\mathrm{km} \cdot \mathrm{h}-1$ para o LAn determinado por meio do ponto de deflexão da FC. Este último se aproxima dos resultados observados no presente estudo, pois os dados coletados apresentaram avaliação da aptidão aeróbia apenas para alguns indivíduos do sexo masculino.

A caracterização e classificação dos indivíduos quanto aos resultados observados para - LAn é complexa devido a outros indicadores serem utilizados com mais frequência para avaliação da aptidão aeróbia $[17,18,23]$. Desta forma, salientamos que uma possível limitação do estudo foram os testes empregados no presente trabalho, pois são medidas indiretas das capacidades físicas investigadas. No entanto, esses protocolos de avaliações têm sido utilizados em outros estudos [18,23-25] e são vantajosos devido a simplicidade e o baixo custo financeiro. Além disso, é importante ressaltar que o objetivo principal do estudo foi estabelecer o perfil dos praticantes dos projetos de extensão.

\section{Conclusão}

O presente estudo demonstrou que os indivíduos praticantes dos projetos de extensão de uma instituição de ensino superior apresentam um baixo nível de aptidão física. Estratégias que estimulem a mudança de hábitos em relação à atividade física podem contribuir para a melhora desse quadro. Campanhas de incentivo à participação em programas de atividade física podem ser realizadas também pelos meios digitais [20], informando sobre os benefícios da prática regular de atividade física e da adoção de hábitos saudáveis para melhorar a qualidade de vida. Ainda mais, ações direcionadas à realização de eventos periódicos e de grande proporção também auxilia no incentivo à prática de esportes e de exercícios físicos, como é o caso de jogos universitários, eventos festivos com a inclusão de danças, atividades ao ar livre, entre outros. É importante destacar que não é função única e exclusiva das instituições de ensino superior melhorar a aptidão física voltada para a saúde e a qualidade de vida desses indivíduos, necessitando, portanto, de parcerias e ações conjuntamente com as instituições públicas. Entretanto, as ações e estratégias sugeridas aqui podem fazer com as instituições de ensino superior forneçam subsídios para a adoção de comportamentos relevantes em relação à saúde.

Portanto, conclui-se que o perfil da aptidão neuromuscular dos indivíduos do presente estudo indica a necessidade de ações e planejamentos de programas que procurem melhorar o nível de aptidão física desses indivíduos. Ainda mais, a caracterização da aptidão aeróbia pode permitir aos profissionais e praticantes, o uso de dados relevantes para a elaboração da prescrição e planejamento dos treinamentos e das ações das instituições de ensino superior.

\section{Referências}

1. Polisseni MLDC, Ribeiro LC. Exercício físico como fator de proteção para a saúde em servidores públicos. Rev Bras Med Esporte 2014;20(4):340-4.

2. Oliveira CS, Gordia AP, Quadros TMB, Campos W. Atividade física de universitários brasileiros: uma revisão de literatura. RAS 2014;12(42):71-7.

3. Hammami A, Randers MB, Kasmi S, Razgallah M, Tabka Z, Chamari K, et al. Effects of soccer training on health-related physical fitness measures in male adolescents. Journal of Sport and Health Science 2018;7(2):169-75. 2018.

4. Yıldızer G, Bilgin E, Korur EN, Novak D, Demirhan G. The association of various social capital indicators and physical activity participation among Turkish adolescents. Journal of Sport and Health Science 2017;7(1):27-33.

5. Roh HT, So WY. The effects of aerobic exercise training on oxidant-antioxidant balance, neurotrophic factor levels, and blood-brain barrier function in obese and non-obese men. Journal of Sport and Health Science 2017;6(4):447-53. 
6. Gadais T, Boulanger M, Trudeau F, Rivard MC. Environments favorable to healthy lifestyles: A systematic review of initiatives in Canada. Journal of Sport and Health Science 2018; 7(1):7-18.

7. He LI, Wei WR, Can Z. Effects of 12-week brisk walking training on exercise blood pressure in elderly patients with essential hypertension: a pilot study. Clin Exp Hypertens 2018;1-7.

8. Castagna C, de Sousa M, Krustrup P, Kirkendall D. Recreational team sports: The motivational medicine. Journal of Sport and Health Science. 2018. https://doi.org/10.1016/j.jshs.2017.12.001

9. Brasil. Parâmetros curriculares Nacionais: Educação Física. Brasília: MEC; 1998.

10. Alves JGB, Montenegro FMU, Oliveira FA, Alves RV. Prática de esportes durante a adolescência e atividade física de lazer na vida adulta. Rev Bras Med Esporte 2005;11:291-4.

11. Paes ST, Marins JCB, Andreazzi AE. Efeitos metabólicos do exercício físico na obesidade infantil: uma visão atual. Rev Paul Pediatr 2015;33(1):122-9.

12. Barbanti VJ. Teoria e prática do treinamento esportivo. São Paulo: Edgard Blücher; 1997.

13. Mann T, Lamberts RP, Lambert MI. Methods of prescribing relative exercise intensity : physiological and pratical considerations. Sports Med 2013;43:613-25.

14. Conconi F, Grazzi G, Casoni I, Guglielmini C, Borsetto C, Ballarin E, et al. The Conconi test: methodology after 12 years of application. Int J Sports Med 1996;17(7):509-19.

15. Nascimento EMF, Silva AEL, Bertuzzi RCM, Kiss MAPDM, Pires FDO. Caracterização da curva da frequência cardíaca durante teste incremental máximo em esteira. Rev Bras Cineantropom Desempenho Hum 2011;13:285-91.

16. Fernandes A. Manual de Avaliação Física. São Paulo: Ícone; 2012

17. Glaner MF, Pires Neto CS, Zinn JL. Diagnóstico da aptidão física relacionada à saúde de universitários. Rev Bras Ativ Fís Saúde 1998;3(4): 35-41.

18. Loch MR, Konrad LM, Santos PDD, Nahas MV. Health-related fitness profile of college students attending physical education classes. Rev Bras Cineantropom Desempenho Hum 2006;8(1):64-71.

19. Freitas Junior RIF. Aptidão Física relacionada à saúde em adultos. Rev Bras Ativ Fís Saúde 1995;1(2):39-48.

20. Corseuil MW, Petroski EL. Baixos níveis de aptidão física relacionada à saúde em universitários. Rev Bras Educ Fís Esporte 2010;24(1):49-54.

21. Brunetta HS, Navarro AC, Frighetto M. Análise do lactato em duas sessões de corrida prescrita através do ponto de deflexão da frequência cardíaca. Revista Brasileira de Prescrição e Fisiologia do Exercício 2013;7(42):522-8.

22. Kjertakov M, Dalip M, Hristovski R, Epstein Y. Prediction of lactate threshold using the modified Conconi test in distance runners. Physiol Int 2016;103(2):262-70.

23. Ribeiro AS. Aptidão física relacionada à saúde em homens e mulheres de 17-26 anos. Rev Bras Ativ Fís Saúde 2013;18(2):197-204.

24. Pereira PEA, Piubelli Carrara VK, Pereira Duarte JM, Fernandes Guerra RL, Azevedo PHSM. The relationship between the heart rate deflection point test and maximal lactate steady state. J Sports Med Phys Fitness 2016;56(5):497-502.

25. Nogueira JAD, Pereira $\mathrm{CH}$. Aptidão física relacionada à saúde de adolescentes participantes de programa esportivo. Rev Bras Educ Fís Esporte 2014;28(1):31-40. 